\title{
Recruiting Injection Drug Users: A Three-Site Comparison of Results and Experiences with Respondent-Driven and Targeted Sampling Procedures
}

\author{
William T. Robinson, Jan M. H. Risser, Shanell McGoy, Adam B. Becker, \\ Hafeez Rehman, Mary Jefferson, Vivian Griffin, \\ Marcia Wolverton, and Stephanie Tortu
}

\begin{abstract}
Several recent studies have utilized respondent-driven sampling (RDS) methods to survey hidden populations such as commercial sex-workers, men who have sex with men (MSM) and injection drug users (IDU). Few studies, however, have provided a direct comparison between RDS and other more traditional sampling methods such as venue-based, targeted or time/space sampling. The current study sampled injection drug users in three U.S. cities using RDS and targeted sampling (TS) methods and compared their effectiveness in terms of recruitment efficiency, logistics, and sample demographics. Both methods performed satisfactorily. The targeted method required more staff time per-recruited respondent and had a lower proportion of screened respondents who were eligible than RDS, while RDS respondents were offered higher incentives for participation.
\end{abstract}

KEYWORDS Injection drug use, Sampling methodology, Hidden populations, Respondent-driven sampling, Targeted sampling.

\section{INTRODUCTION}

Injection drug users (IDU) remain an important population in the study of HIV/ AIDS. The Centers for Disease Control and Prevention (CDC) estimates that $31.8 \%$ of cumulative male U.S. AIDS cases through 2004 and $40.7 \%$ of female cases were exposed through injection drug-use or male-to-male sexual contact and injection drug use. ${ }^{1}$ Unfortunately traditional sampling methods are inadequate when the population of interest consists of individuals who perform illegal or stigmatized behaviors. Injection drug users are one such population. Traditional probabilitybased sampling methods require the development of a sampling frame enumerating the entire population. This is prohibitive for these 'hidden' populations because their size is unknown, and their members are often reluctant to participate in studies due to legal issues or social stigmatization. ${ }^{2,3}$

Robinson is with the HIV/AIDS Program, Louisana Office of Public Health, 1010 Common St. 11th floor, New Orleans, LA 70112, USA; Risser is with the University of Texas School of Public Health, Houston, TX, USA; McGoy and Griffin are with the Michigan Department of Community Health, Detroit, MI, USA; Becker is with the Louisiana Public Health Institute, New Orleans, LA, USA; Rehman and Wolverton are with the Houston Department of Health and Human Services, Houston, TX, USA; Jefferson is with the Tulane University, New Orleans, LA, USA; Tortu is with the Louisiana State University Health Sciences Center School of Public Health, New Orleans, LA, USA.

Correspondence: William T. Robinson, Louisana Office of Public Health, 1010 Common St. 11th floor, New Orleans, LA 70112, USA. (E-mail: brobinso@dhh.la.gov) 
Because of these difficulties, a number of alternative sampling methods have historically been employed to study hidden populations, including injection drug users. ${ }^{4}$ These methods have included convenience sampling, time/space or venuebased sampling and chain-referral methods. Chain-referral or "snow-ball" sampling ${ }^{5,6}$ methods involve selection of an initial set of members of the target population, or 'seeds', who provide information on, or referrals for, subsequent waves of respondents. The number of waves and potential referrals, as well as the seed selection methods, may vary. Versions of these methods have proven successful at quickly and easily identifying members of hidden populations. ${ }^{7-9}$ However, results obtained are thought to be biased by the initial seed selection method as well as characteristics of the respondents' social network and have generally been classified as convenience samples. ${ }^{6,10,11}$

Another method proposed by Watters and Biernacki ${ }^{3}$ known as targeted sampling (TS) has had some success in recruiting injection drug users. ${ }^{12}$ Targeted sampling requires extensive ethnographic and formative research to describe the area and population of interest and to identify appropriate locations within that area for inclusion in a sampling plan. ${ }^{13}$ Existing secondary data sources are also reviewed to further describe the target population and geographic areas of interest. Qualitative data in the form of focus groups and in-depth interviews with key informants may be conducted to gain a more complete understanding of the population of interest as well as to identify possible specific locations for sampling. These locations are then systematically mapped and ethnographic observations are conducted. Based on all of this information a sampling frame of locations is developed and weighted by the density of observed indicators. Locations are randomly selected and recruitment is conducted within the selected locations. Targeted sampling, as well as other time-space or venue-based sampling methods, may be biased to the extent that members of the population of interest do not attend the venues or areas that have been identified.

Heckathorn ${ }^{10,14,15}$ has recently proposed a modified chain-referral method called respondent-driven sampling (RDS) as a promising strategy in surveying hidden populations. This approach retains the advantages of chain-referral sampling in terms of ease of respondent identification and recruitment while allowing population estimates and inferences to be made. ${ }^{10,15}$ Initial seeds are identified from known members of the population or through key informants. Information about the respondent's social network is collected, and seeds are provided with a set number of coupons for distribution to recruits within their own network. Eligible recruits are interviewed and provided coupons for distribution to the next 'wave'. Recruitment continues in this way until the desired sample size is reached. Network information is then used to derive asymptotically unbiased population estimates for variables of survey interest.

RDS has been shown to be a useful strategy for the recruitment of drug users. $^{16-18}$ To date, however, no direct comparison of RDS with other sampling methods has been made in terms of the ability to recruit hidden populations such as IDUs, the "representativeness" of sample characteristics, and the practicality of administration. ${ }^{2,4}$ The objective of the current study is to compare and contrast RDS and TS methods on demographics, recruitment efficiency, and logistic concerns.

\section{MATERIALS AND METHODS}

Three sites in large Metropolitan Statistical Areas (MSA) (Detroit, Houston and New Orleans) that participated in the CDC-funded National HIV Behavioral 
Surveillance among injecting drug users (NHBS-IDU) conducted a pilot investigation comparing RDS and TS. Each site assessed the feasibility of recruiting at least 100 eligible IDUs utilizing RDS and TS methods over an 8-week period in late 2004. What follows is a brief presentation of the strategies used by each site to locate and recruit potential respondents using RDS and TS methods.

\section{Targeted Sampling}

Formative Research Extensive ethnographic and formative research was conducted at all sites prior to sampling. Each site completed a thorough review of secondary data sources dealing with the IDU population. Data were compiled and synthesized from several sources including number of persons living with HIV/AIDS who have IDU as a reported mode of exposure, archival census data and findings from previous published and unpublished research.

A total of 81 in-depth interviews were conducted with individuals who had knowledge of local injection drug-use communities. Those interviewed included local HIV prevention personnel and community planning group members as well as law enforcement representatives and current or former injectors. Ten focus groups with a total of 78 injection drug users were also conducted in order to provide specific knowledge about local injection habits and behaviors. These structured interviews were designed to provide a fuller understanding of the injection community at large as well as to solicit locations for possible sampling areas. Sites also utilized information on areas that had been previously identified by ethnographers with knowledge of the local IDU community or had been used in other local IDU studies.

Area Identification and Recruitment Field observations of the possible sampling areas were systematically conducted at varying times of day and week over the course of 5 months (March-July 2004). Field staff conducted ethnographic observations within each area and recorded any IDU indicators that were present. IDU indicators included physical objects such as used syringes, baggies, balloons or injection works/equipment as well as behavioral indicators such as copping activity, loitering or commercial sex work. Sites used a combination of ethnographic research, secondary data analysis, key informant interviews, and field observations to form expected yield ratings to identify areas where injection drug use was likely to occur and where potential survey respondents could be recruited.

Based on highest expected yield rating, Detroit and Houston identified four and ten sampling areas, respectively, within seven ZIP codes. For these sites line based enumeration was then used to approach possible respondents. New Orleans identified 35 sampling areas within 16 ZIP codes of high, medium and low expected yield. These ZIP codes were stratified by the expected yield, and order of ZIP codes was then randomly selected without replacement at the beginning of each 2 -week period. Interviewers approached all individuals within each of the prescribed three block sampling areas within each ZIP code.

\section{Respondent-Driven Sampling}

Seed Selection and Recruitment Possible seeds were identified through key informant referrals and street outreach. Seeds were purposefully selected to maximize diversity on race, sex and drug of choice. Each seed was screened, and eligible seeds 
were interviewed using the NHBS-IDU instrument and provided with a $\$ 20$ cash value incentive. Each initial seed was provided with three recruitment coupons and instructed to give them to individuals they knew injected drugs. Seeds were also informed that they would be compensated with $\$ 10$ cash value for each person that they recruited. Peer-recruited respondents returning these coupons were then screened for eligibility, interviewed and provided with three coupons they were instructed to give to someone who they knew injected drugs. They were told that they would be compensated $\$ 10$ for each person they recruited. A specialized coupon tracking system database was used to track referrals and facilitate incentive provision.

Analysis All RDS participants were asked to provide information about the size and characteristics of their social networks from which they would draw potential recruits. Specifically, each participant was asked to report the number, sex and race/ ethnicity of injectors they knew and had seen in the past 6 months. Relationship of the respondent to the recruiter (e.g., friend, injection partner, sex partner etc.) was also assessed. This information along with data from the coupon tracking system was analyzed using Respondent-Driven Sampling Analysis Tool (RDSAT) software (RDSAT (computer version). Version 5.4.0. Ithaca, New York: Volz E, Heckathorn $\mathrm{DD} ; 2005)$ to provide estimated population proportions and confidence intervals for age, sex and race/ethnicity variables.

\section{Participants}

All participants provided informed consent and were carefully screened for eligibility criteria. Eligibility criteria for study participation were that the respondent was at least 18 years of age, was resident of the study MSA and had injected illicit drugs within the past 12 months.

Targeted Sampling In Detroit 273 individuals were approached during TS. Two hundred twenty one individuals agreed to participate and were screened for eligibility. Of TS screened participants, 54\% $(n=120)$ were found to be eligible and completed the survey.

In Houston 174 individuals were approached during TS. One hundred forty eight respondents agreed to participate and were screened for eligibility. Of TS screened participants, $65 \%(n=97)$ were found to be eligible and completed the survey.

In New Orleans 560 individuals were approached during TS. Three hundred eightynine respondents agreed to participate and were screened for eligibility. Of TS screened participants, $35 \%(n=137)$ were found to be eligible and completed the survey.

Respondent-Driven Sampling Six seeds were approached in Detroit for participation, and three of those individuals completed the survey and agreed to be seeds. One hundred sixty-nine recruitment coupons were distributed by Detroit during RDS over nine waves of recruitment; 106 of those individuals returned and were screened for eligibility. Of RDS screened participants, $96 \%(n=102)$ were found to be eligible and completed the survey.

Thirteen seeds were approached in Houston for participation, and one additional seed was recruited after being interviewed in the targeted sampling component. Of the 14 seeds recruited, 1 was found to be ineligible. One hundred sixty-eight recruitment coupons were distributed during RDS over seven waves of recruitment; 62 of those individuals returned and were screened for eligibility. Of RDS screened participants, $77 \%(n=48)$ were found to be eligible and completed the survey. 
Ten seeds were approached in New Orleans for participation, and two of those individuals completed the survey and agreed to be seeds. Three hundred sixteen recruitment coupons were distributed during RDS over 19 waves of recruitment; 133 of those individuals returned and were screened for eligibility. Of RDS screened participants, $88 \%(n=118)$ were found to be eligible and completed the survey.

\section{Materials}

The standardized NHBS-IDU questionnaire was administered to all eligible participants. This 68-item questionnaire on HIV risk behaviors, requiring approximately $40 \mathrm{~min}$, includes items on demographic characteristics, drug use, sexual risk behaviors, access to and use of health care and utilization of HIV prevention services. Variables of interest for this study included respondent demographic information, time required for survey completion, and cost of each survey.

\section{RESULTS}

\section{Sample Characteristics}

Demographic characteristics of the samples obtained for each site by method are presented in Tables 1, 2, and 3. Local HIV surveillance data on persons living with HIV/AIDS who have injection drug use as a reported mode of exposure are presented for comparison purposes only. Chi-square tests indicated no significant association of sampling method and gender for any site (Detroit: $\chi_{1}^{2}=0.96, P=$ 0.33; Houston: $\chi_{2}^{2}=1.93, P=0.38$; New Orleans: $\chi_{2}^{2}=2.93, P=0.23$ ). No significant association between age and sampling method was found (Detroit: $\chi_{3}^{2}=2.27, P=0.52$; Houston: $\chi_{2}^{2}=4.0, P=0.14$; New Orleans: $\chi_{3}^{2}=4.3, P=$ $0.23)$. Two sites showed significant association between race/ethnicity and sampling method (Detroit: $\chi_{3}^{2}=2.82, P=0.42$; Houston: $\chi_{3}^{2}=11.2, P=0.01$; New Orleans: $\left.\chi_{2}^{2}=38.23, P<0.0001\right)$.

TABLE 1. Demographic characteristics of Detroit IDUs obtained through RDS and TS, and persons living with HIV/AIDS who are injection drug users (PLWH/A-IDU)

\begin{tabular}{|c|c|c|c|c|c|c|}
\hline \multirow[b]{2}{*}{ Race/ethnicity } & \multicolumn{2}{|c|}{ TS } & \multicolumn{2}{|c|}{ RDS } & \multirow[b]{2}{*}{ RDS pop. estimates, \% (95\% Cl) } & \multirow[b]{2}{*}{ PLWH/A-IDU, (\% } \\
\hline & $N$ & $\%$ & $N$ & $\%$ & & \\
\hline African American & 101 & 94 & 96 & 96 & - & 91 \\
\hline White & 0 & 0 & 1 & 1 & - & 5 \\
\hline Hispanic & 1 & 1 & 1 & 1 & - & 3 \\
\hline Other & 6 & 6 & 2 & 2 & - & 0 \\
\hline \multicolumn{7}{|l|}{ Sex/gender } \\
\hline Male & 74 & 69 & 71 & 71 & $63(52-75)$ & 61 \\
\hline Female & 34 & 31 & 24 & 24 & $37(25-48)$ & 39 \\
\hline \multicolumn{7}{|l|}{ Transgender } \\
\hline Age & & & & & & \\
\hline $18-34$ & 2 & 1 & 2 & 2 & $0.1(0.2-0.7)$ & 4 \\
\hline $35-44$ & 24 & 23 & 20 & 20 & $17(7-27)$ & 22 \\
\hline $45-54$ & 65 & 60 & 54 & 54 & $51(40-67)$ & 47 \\
\hline $55+$ & 17 & 16 & 24 & 24 & $32(16-43)$ & 27 \\
\hline
\end{tabular}


TABLE 2. Demographic characteristics of Houston IDUs obtained through RDS and TS, and persons living with HIV/AIDS who are injection drug users (PLWH/A-IDU)

\begin{tabular}{|c|c|c|c|c|c|c|}
\hline \multirow[b]{2}{*}{ Race/ethnicity } & \multicolumn{2}{|c|}{ TS } & \multicolumn{2}{|c|}{ RDS } & \multirow[b]{2}{*}{ RDS pop. estimates, $\%(95 \% \mathrm{Cl})$} & \multirow[b]{2}{*}{ PLWH/A-IDU, (\%) } \\
\hline & $N$ & $\%$ & $N$ & $\%$ & & \\
\hline African American & 42 & 52 & 27 & 45 & - & 69 \\
\hline White & 22 & 28 & 14 & 23 & - & 20 \\
\hline Hispanic & 10 & 12 & 19 & 32 & - & 10 \\
\hline Other & 6 & 7 & 0 & 0 & - & 1 \\
\hline \multicolumn{7}{|l|}{ Sex/gender } \\
\hline Male & 53 & 67 & 46 & 77 & $76(45-96)$ & 56 \\
\hline Female & 20 & 25 & 12 & 20 & $24(4-55)$ & 44 \\
\hline Transgender $^{\mathrm{a}}$ & 6 & 8 & 2 & 3 & & \\
\hline \multicolumn{7}{|l|}{ Age } \\
\hline $18-34$ & 26 & 34 & 12 & 20 & $14(0.1-31)$ & 17 \\
\hline $35-44$ & 22 & 28 & 25 & 41 & $41(33-86)$ & 40 \\
\hline $45+$ & 30 & 39 & 24 & 40 & $45(5-60)$ & 43 \\
\hline
\end{tabular}

${ }^{\mathrm{a}}$ Female and transgender categories were combined for purposes of Houston's RDS analyses.

Also presented are the population estimates and confidence intervals obtained by RDSAT. No RDS population estimates were calculated for race/ethnicity for Detroit or Houston due to lack of cross-group recruitment (e.g., only African Americans were recruiters of other race/ethnicities in Detroit). For each RDS estimate presented, equilibrium was achieved.

\section{Cost Effectiveness}

Across all sites $47 \%$ of TS-screened respondents were found to be eligible IDUs, while $89 \%$ of screened respondents in RDS were eligible IDUs. TS required an average of $214 \mathrm{~h}$ performing ethnographic observation and mapping plus an

TABLE 3. Demographic characteristics of New Orleans IDUs obtained through RDS and TS, and persons living with HIV/AIDS who are injection drug users (PLWH/A-IDU)

\begin{tabular}{|c|c|c|c|c|c|c|}
\hline \multirow[b]{2}{*}{ Race/ethnicity } & \multicolumn{2}{|c|}{ TS } & \multicolumn{2}{|c|}{ RDS } & \multirow[b]{2}{*}{ RDS pop. estimates, \% $(95 \% \mathrm{Cl})$} & \multirow[b]{2}{*}{ PLWH/A-IDU, (\%) } \\
\hline & $N$ & $\%$ & $N$ & $\%$ & & \\
\hline African American & 125 & 89 & 67 & 58 & 77 (64-90) & 65 \\
\hline White & 5 & 4 & 34 & 29 & $20(9-34)$ & 33 \\
\hline Hispanic & 1 & 1 & 2 & 2 & $3(0.1-3)$ & 2 \\
\hline Other ${ }^{\mathrm{a}}$ & 9 & 6 & 13 & 11 & & 0 \\
\hline \multicolumn{7}{|l|}{ Sex/gender } \\
\hline Male & 119 & 85 & 94 & 81 & $74(58-88)$ & 76 \\
\hline Female & 19 & 14 & 22 & 19 & $26(12-42)$ & 24 \\
\hline Transgender & 2 & 1 & & & & \\
\hline \multicolumn{7}{|l|}{ Age } \\
\hline $18-34$ & 37 & 26 & 34 & 29 & $27(15-43)$ & 16 \\
\hline $35-44$ & 44 & 31 & 26 & 22 & $27(12-44)$ & 35 \\
\hline $45-54$ & 40 & 29 & 44 & 38 & $39(21-57)$ & 37 \\
\hline $55+$ & 19 & 14 & 12 & 10 & $7(2-13)$ & 12 \\
\hline
\end{tabular}

${ }^{\mathrm{a}}$ Hispanic and other race/ethnicities were combined for purposes of New Orleans' RDS analyses. 
average of approximately $2 \mathrm{~h}$ and $9 \mathrm{~min}$ of staff time locating, screening and interviewing each recruit (total $=4 \mathrm{~h}$ and $\min$ per recruit) while RDS required approximately $1 \mathrm{~h}$ and 13 min per recruit. TS methods provided each participant with an average of $\$ 20$ in incentives, while RDS recruits received an average of $\$ 26.68$. RDS incentives did not total $\$ 50$ for every participant because some respondents did not produce the maximum of three recruits, and some recruiters did not return to receive the added incentives for recruitment efforts.

\section{Logistics}

Staffing requirements were similar for both methods, and sites were able to use the same staff to conduct RDS and TS concurrently. Some differences were evident as a result of method selection. Targeted sampling was conducted in the field while RDS was usually conducted from a single storefront. Interviewers reported that this led to a more controlled environment when using RDS. Increased privacy, comfort and perceptions of safety were reported, while distraction level decreased. Weather was also more of a concern with TS while interviewing outdoors.

\section{DISCUSSION}

The use of multiple sources of ethnographic data was beneficial in identifying highyield TS recruitment areas because areas with the highest number of eligible recruits were often not necessarily areas with obvious drug use indicators but areas identified through HIV surveillance or key-informant interviews. However, even with good ethnographic information, many areas that were expected to be highyield were not, and less than half of all individuals approached were found to be eligible. This led to low interviewer morale in Detroit, where the interviewers were paid per interview completed. It also led to some bias in recruitment in Houston, where interviewers made the decision to recruit only individuals who looked like injection drug users on one particularly unsuccessful recruitment day.

The sample characteristics of those recruited through TS may not reflect the general IDU population but rather the population found during the recruitment times. New Orleans included low density areas and randomized area selection in the hopes of assembling a diverse sample, reflective of the general IDU population. However, this did not happen. The sample characteristics were reflective of the population found during recruitment times, and the sample may have missed less visible injectors, such as those with steady day time employment or child care responsibilities and those who remain indoors during the day or only come out at night. Houston and Detroit had similar problems. One solution might be to extend recruitment hours into the night or start very early in the morning, but this would further increase security concerns.

TS offered less control in the working environment, compared to RDS. Safety was a concern: The interviewers tended to spread out in the targeted area and the interviewing process drew the attention of others. Groups often gathered to inquire about the interview and the incentive, heightening security concerns. Weather was also an important factor in recruitment success, as fewer individuals were on the streets during hot, cold, or rainy days, and the spring and summer of 2005 was particularly noteworthy, weather-wise. Overall the RDS storefront was preferable to conducting TS outdoors. Participants often scheduled interview appointments and came to a central location for their interviews. Adequate staffing and office space were essential. It was found to be best to have a general waiting area, private 
interview spaces for each interviewer, and an area for obtaining participants' coupon information and providing incentives. In addition, all sites concurred that a single staff person to monitor the flow of participants entering, waiting, and exiting the facility would have provided even more control of the environment.

RDS success depends largely on the effective recruitment of seeds, an interconnectedness of injection drug use networks, and the willingness of seeds and their recruits to travel to an interview location. Community partnerships were also important in the recruitment of successful seeds. In Detroit, seeds were recruited from two needle exchange locations in the city at the recommendation of staff familiar with the individuals' ability to recruit others. This worked well in Detroit, where three of six recruited seeds led to the recruitment of 106 eligible IDUs.

In Houston, the initial seeds were recruited from outreach workers who were conducting other research studies in drug-using populations. This was problematic, as some of these initial seeds were not actually linked to the injection drug using community but rather spent their days recruiting participants for these other research projects. These "research hustler" seeds were often very good at recruiting individuals (for money), just not at recruiting eligible individuals. This resulted in very short referral chains since few of their recruits were eligible.

In New Orleans, staff were only able to successfully recruit two seeds. Eight others identified through key informants agreed to participate but never came to the field location to be interviewed. The two seeds that were interviewed represented the two subgroups of the overall sample, as described in the formative research. One seed was an older African American male, the other a younger white female. The race/ethnicity and age differences between the New Orleans RDS sample $(29 \%$ white, $19 \%$ under 25$)$ and the TS sample (4\% white, 5\% under 25$)$ may have reflected the characteristics of the two seeds' networks rather than methodological differences.

Based on lessons learned in this pilot, when NHBS-IDU shifted to full implementation, the sites made several adjustments to the RDS protocols. In New Orleans, prior to the discontinuation of the project due to Hurricane Katrina, seeds were recruited from among participants who were known to interviewers from the TS pilot and who encouraged others to be interviewed. Second, two field offices were established; one was close to downtown and easily accessible by public transportation and the other was near a large public housing project in an area that yielded high eligibility rates in TS. Finally, transportation tokens were purchased and made available to participants who came to the field office in an attempt to ease the burden of travel on respondents. In Houston, successful seeds were individuals the interviewers/outreach workers found during the formative work for the targeted pilot. These were influential individuals or gatekeepers within small communities of injection drug users, often the individuals who were first to approach staff and inquire why interviewers were entering their geographic space.

Discontinuing RDS recruitment required some special considerations. While targeted sampling may easily stop on any given date or after a pre-determined number of participants have been recruited, the coupons distributed to potential RDS subjects represent a commitment on the part of the researcher. It was therefore important to carefully decrease the number of coupons distributed and shorten the coupon validity period so that recruitment would continue even as the end of the study date approached. Ending the distribution of coupons too early may result in not meeting the recruitment goal (in this case 100 participants), while ending it too late may lead to a disgruntled public. 
Overall, both RDS and TS proved effective in recruiting IDUs in the three pilot MSAs. Although no claim as to the actual representativeness of the obtained samples is made, both methods yielded samples that were similar in terms of many demographic characteristics (such as age, race and sex) as well as drug of choice (not presented here). Demographic results of the samples from both methods also compared favorably to each city's Census and HIV surveillance data from injection drug users. All three sites were able to reach the goal of 100 respondents in TS. Issues with initial seed selection and time constraints most likely resulted in the failure of one site to recruit 100 respondents with RDS. Total cost efficiency for the two methods was also comparable. Extensive formative research and low screenedto-eligible respondent ratios resulted in larger person-hour expenditures for TS, while RDS required higher incentive payouts to participants. Logistical concerns did differ considerably between the two methods. While RDS provided a more controlled interview environment, recruitment success seemed to be affected by the ability to identify quality seeds. Quality of ethnographic assessment and size and cultural qualities of the MSAs also affected targeted sampling success. While more research is needed in assessing the methodological impact of choice of sampling method in identifying hidden populations, these experiences should provide researchers some insight into the some of the practical strengths and weaknesses of these two methods.

\section{ACKNOWLEDGEMENTS}

This study was supported in part by grants PA 04 - 017 from the Centers for Disease Control and Prevention. The authors wish to thank two anonymous reviewers for their comments as well as Abu Abdul-Quader and the CDC-National HIV Behavioral Surveillance project staff for guidance and technical assistance.

\section{REFERENCES}

1. Centers for Disease Control and Prevention. HIV/AIDS Surveillance Report, 2004. U.S. Department of Health and Human Services, Centers for Disease Control and Prevention. 2005; Vol. 16.

2. Magnani R, Sabin K, Saidel T, Heckathorn D. Review of sampling hard-to-reach and hidden populations for HIV surveillance. AIDS. 2005;19(2):S67-S72.

3. Watters JK, Biernacki P. Targeted sampling: options for the study of hidden populations. Soc Probl. 1989;36(4):416-430.

4. Semaan S, Lauby J, Liebman J. Street and network sampling in evaluation studies of HIV risk-reduction interventions. AIDS Rev. 2002;4:213-223.

5. Coleman JS. Relational analysis: the study of social organization with survey methods. Human Organ. 1959;17:28-36.

6. Goodman L. Snowball sampling. Ann Math Stat. 1961;32(1):148-170.

7. Erickson BH. Some problems of inference from chain data. Sociol Method. 1979;10:276-302.

8. Klovdahl A. Urban social networks: some methodological problems and possibilities. In: Kochen M, ed. The Small World. Norwood, NJ. ABLEX 1989;176-210.

9. Dodds PS, Muhamad R, Watts DS. An experimental study of search in global social networks. Science. 2002; 301(5634): 827-829.

10. Salganik MJ, Heckathorn DD. Sampling and estimation in hidden populations using respondent-driven sampling. Sociol Method. 2004;34:193-239. 
11. Biernacki P, Waldorf D. Snowball sampling: problems and techniques of chain referral sampling. Sociol Methods Res. 1981;10(2):141-163.

12. Kral AH, Bluthenthal RN, Booth RE, Watters JK. HIV seroprevalence among streetrecruited injection drug and crack cocaine users in 16 U.S. municipalities. Am J Public Health. 1998;88(1):108-114.

13. Bluthenthal RN, Watters JW. Multimethod research from targeted sampling to HIV risk environments. In: Lambert EY, Ashery RS, Needle RH, eds. Qualitative Methods in Drug Abuse and HIV Research. National Institute on Drug Abuse Research Monograph. 1994; Vol. 157, pp 212-230.

14. Heckathorn DD. Respondent-driven sampling: a new approach to the study of hidden populations. Soc Probl. 1997;44(2):174-199.

15. Heckathorn DD. Respondent-driven sampling II: deriving valid population estimates from chain referral samples of hidden populations. Soc Probl. 2002;49(1):11-34.

16. Heckathorn DD, Semaan S, Broadhead RS, Hughes JJ. Extensions of respondent-driven sampling: a new approach to the study of injection drug users aged 18-25. AIDS Behav. 2002;6(1):55-67.

17. Wang J, Carlson RG, Falck RS, Siegal HA, Rahman A, Li L. Respondent-driven sampling to recruit MDMA users: a methodological assessment. Drug Alcohol Depend. 2005;78:147-157.

18. Abdul-Quader AS, Heckathorn DD, McKnight C, et al. Effectiveness of respondentdriven sampling for recruiting drug users in New York City: findings from a pilot study. $J$ Urban Health. 2006;83(3):459-476. 